\title{
Knowledge-based classification of ill-defined categories
}

\author{
GLENN V. NAKAMURA \\ University of Illinois, Champaign, Illinois
}

\begin{abstract}
In this paper, I report an exploratory study which investigated the role that prior knowledge plays in influencing classification learning. Under neutral or knowledge-imposing instructions, subjects learned to classify exemplars into categories that either were or were not linearly separable. Linearly separable categories are those categories whose members can be correctly classified based on an additive summation of weighted attribute information. Following category learning, the subjects were given transfer tests. A major finding was that knowledge facilitated the learning of linearly separable categories but interfered with the learning of not linearly separable categories. Quantitative analyses revealed that the knowledge facilitated category learning of the linearly separable categories by influencing the storage and reliance on both prototypical and exemplar information.
\end{abstract}

There are many ways in which an individual can learn about a category. Past research has examined the cognitive contributions of hypotheses, rules, and strategies to category learning; however, the use of one's previously acquired knowledge in category learning has not received a great deal of research attention. The present study explored the role that recently acquired knowledge plays in classification learning. Before considering the details of the present study, a brief review of past work on category learning is in order.

There have been two views concerning the structure of categories. The traditional view (see Bourne, 1966) argued that categories are well defined in that all category members contain criterial attributes that are singly necessary and jointly sufficient for determining class membership. According to this view, all members should be equally representative of a category, and there should not be any doubt about whether an instance is or is not a member of the category.

More recently, it has been argued that most categories are ill-defined, that category membership is based on

The research reported in this article was based on a master's thesis submitted to the University of Illinois Graduate College in order to partially fulfill the requirements for a master's degree in psychology. The research reported here was supported by National Institute of Mental Health Grant MH 32370 to Douglas L. Medin, and by a Graduate College Fellowship to the author. I am grateful to Douglas Medin for supporting this research and for providing invaluable advice and encouragement during all phases of this research. Also, this research benefited from discussions with members of the Douglas Medin Research Group. Thanks are due to Timothy Hajek for his excellent assistance in helping with the data collection, and to Mark Klein for the use of his computer program that presented the category learning task and transfer task. Finally, thanks are due to William Brewer, Charles Clifton, Norman Slamecka, and an anonymous reviewer for providing very helpful comments on one or two drafts of this paper. All correspondence regarding this article should be addressed to G. Nakamura, Department of Psychology, University of Illinois, 603 E. Daniel, Champaign, IL 61820. characteristic attributes, and that category members vary in the degree to which they possess these characteristic attributes (Rosch \& Mervis, 1975). Under this view, category boundaries are vague, and category membership is not an all-or-none affair (McCloskey \& Glucksberg, 1978; Rosch, 1975). Decisions regarding class membership appear to be based on the typicality gradients of class members (Smith, Shoben, \& Rips, 1974). Typicality of an exemplar reflects the number of characteristic features that an exemplar shares with other class members (e.g., family resemblance, Rosch \& Mervis, 1975).

How are individuals able to acquire and use categories that are ill-defined? Research directed at this question has been conducted within one of two theoretical orientations, each of which postulates a particular type of representation as being at the core of classification learning. One major view argues that experience with exemplars of a category leads to the formation of a prototype that serves as the basis for classification performance (Homa \& Vosburgh, 1976; Posner \& Keele, 1968). The prototype is assumed to contain the average or modal features that are derived from experience with exemplars of the category. The degree of similarity between an exemplar and its prototype, as well as its similarity to the prototypes of the contrast categories, is thought to be the key factor controlling the ease in classification. Similarity to the prototype is determined by the number of feature matches that occur between the exemplar and the prototype, with the associated weights (e.g., cue validity, distance, salience, or similarity) for shared feature matches being combined in an independent, additive manner (Franks \& Bransford, 1971; Reed, 1972; see Smith \& Medin, 1981, for a review of prototype models). Thus, an exemplar's degree of similarity to a prototype is reflected by the sum of the weighted attribute information.

An alternative view of category learning does not assume that a prototype mediates classification, but rather 
that classification is assumed to be based on how similar the exemplar to be classified is to other stored exemplars (Brooks, 1978; Hintzman \& Ludlam, 1980; Medin \& Schaffer, 1978; Reitman \& Bower, 1973). According to most exemplar models (e.g., the context model of Medin $\&$ Schaffer, 1978), the key factor governing the ease of classification is how similar the exemplar to be classified is to stored exemplars of one category versus the contrast category. According to the context model, the similarity between any two exemplars is determined by the product of their multiplied attribute similarity values. Thus, high similarity of exemplars within a category should facilitate classification to a greater extent than does high similarity of exemplars to other exemplars in the contrast category (see Smith \& Medin, 1981, for a review of exemplar models).

It has been difficult to find predictions that discriminate between various prototype and exemplar models. For example, Reed's (1972) average-distance model is an exemplar model that uses an additive rule to determine the average distance of the exemplar to be classified from all stored exemplars. Because the average-distance model uses an additive combination rule, it is quite difficult to discriminate between predictions made by it and competing prototype models, which also assume that an additive rule is employed.

One way of distinguishing between classification models is to focus on how the information entering into category judgments is being combined. Medin and Schaffer (1978) have divided classification models into two general classes: independent-cue models and relational-coding models. According to Medin and Schaffer, independentcue models assume that category judgments are based on an additive rule involving an independent, additive summation of weighted attribute information. Also, the additive rule of independent-cue models implies that the addition of this type of rule would not be sensitive to detecting configural information, such as correlated attributes comprising exemplars of a category. Prototype models and the average-distance model can be considered as independent-cue models. In contrast, relational-coding models assume that classification is based on combinations of attribute information. Thus, relational-coding models are sensitive to configural information, such as correlated attributes existing among members of a category (Medin, 1983; Medin, Altom, Edelson, \& Freko, 1982). The context model is an example of a relational-coding model.

There have been a number of studies that have conducted empirical tests between independent-cue models and relational-coding models (e.g., Elio \& Anderson, 1981; Medin et al., 1982; Medin, Dewey, \& Murphy, 1983; Medin \& Schaffer, 1978; Medin \& Schwanenflugel, 1981; Medin \& Smith, 1981). Of importance here was a study by Medin and Schwanenflugel (1981), who tested predictions between independent-cue and relational-coding models by examining classification learning of linearly separable categories and of not linearly separable categories. Linearly separable categories are those categories whose members can be correctly assigned to their respective categories based on a linear-discriminant function that additively combines the weighted attribute information. In contrast, not linearly separable categories exist when there is no way of weighting the attributes so that all members can be assigned to their correct categories based on a summation of weighted attribute information. Independent-cue models imply that the categories should be linearly separable, because these models assume that classification is based on a weighted summation of attribute information, which should lead to the correct classification of all exemplars. In contrast, relational-coding models do not assume that the categories need to be linearly separable, because classification is based on how similar the instance to be classified is to other stored instances of each category.

Medin and Schwanenflugel (1981) predicted that, if attributes were being weighted and additively summed, then the linearly separable categories should have been the easiest to learn. Across a range of learning strategies, stimuli, and category size, there was never any evidence that the linearly separable categories were easier to learn than the not linearly separable categories. In addition, these investigators found that the not linearly separable categories were actually easier to learn than the linearly separable categories, when the former categories contained more exemplars that were highly similar to other category members than to category members of the opposing category. This finding is consistent with relational-coding models.

The Medin and Schwanenflugel (1981) study represents the first attempt to test between independent-cue and relational-coding models by examining the learning of linearly separable and not linearly separable categories. Based on their study, it does not appear that an independent, additive summation of attribute information is used in classification. This conclusion poses a problem specific to prototype models and more generally to independentcue models. Also, the findings of Medin and Schwanenflugel suggest that relational-coding models, such as the context model, might be more plausible approaches to classification learning, because the models' multiplicative rules capture what individuals appear to be doing: classifying on the basis of exemplar similarity.

However, these tentative conclusions arising from this initial study of linear separability may be further qualified in light of understanding how other category-relevant variables affect the storage and reliance on prototypical and exemplar-level information involved in classification. One important class of variables in classification learning has been the role of procedural variables (e.g., rules or learning strategies), which affect the storage and reliance on different levels of category information (prototype and exemplar information) in classification (Medin, Altom, \& Murphy, 1984; Medin \& Smith, 1981).

At the present time, there is a shortage of research that examines how previously acquired knowledge could function as a procedural variable in influencing the storage and reliance on different levels of category information involved in classification (see Medin \& Smith, 1984; Mur- 
phy \& Medin, 1985; Wattenmaker, Dewey, Murphy, \& Medin, 1984a, 1984b, 1984c, for a discussion of knowledgebased classification). Perhaps with the appropriate prior knowledge, the linearly separable categories would be easier to learn, because the knowledge might guide the learner to store and use both prototypical and exemplar-level information in classification. The present study addressed how experimentally imposed knowledge affects the storage of different levels of category information, which in turn govern classification learning of linearly separable and of not linearly separable categories.

The basic experimental design in the present study involved a 2 category structure (linearly separable vs. not linearly separable categories) $\times 2$ instructional set (standard vs. theory instructions) between-subjects design. The two-category structures used in the present study are schematically represented in Table 1 . The top half of Table 1 shows the linearly separable (LS) categories and their respective exemplars; the lower half of the table shows the not linearly separable (NLS) categories and their associated exemplars.

The exemplars composing the LS and NLS tasks were descriptions of hypothetical flowers. A particular flower description is abstractly represented by 0 s and $1 \mathrm{~s}$, which code for one of two possible values on each of the four flower dimensions (D1, D2, D3, and D4). These dimensions correspond to color, petal shape, smell, and habit. A value of 0 on dimensions, D1, D2, D3, and D4, correspondingly indicates the attribute values of bright in color, wide petals, fragrant, and open during the day. A value of 1 on dimensions, D1, D2, D3, and D4, denotes the attribute values of dull in color, narrow petals, odorless, and closed during the day, respectively. Thus, for example, flower description A2 (1010) in Category A of the LS task would be a flower that is dull in color, has wide petals, is odorless, and is open during the day.

One way to see that the categories in the top half of the table are LS is to focus on the first three dimensions. All exemplars in both categories can be correctly classified by placing more weight on the first three dimensions. In contrast, notice that, for the NLS task, there is no way of weighting the dimensions such that every exemplar can be correctly classified. For example, exemplars A1 and
B3 have more attribute values characteristic of the opposing category. Thus, placing more weight on D1 would allow exemplar A1 to be correctly classified, but such heavy weighting on D1 would result in exemplar B3 being erroneously classified as a member of Category A.

The LS and NLS categories also differed in terms of the number of cases of high similarity that existed within as well as between categories. A case of high similarity can be defined when two exemplars differ only on the value of one dimension. In the LS task, there were two cases of high similarity within categories (1011 and 1010, 1001 and 0001 ) and five cases of high similarity between categories (1011 and 1001, 1010 and 0010, 1101 and 1001,0110 and 0010,0110 and 0100 ). In contrast, in the NLS task, there were four cases of high similarity within categories (1000 and 1010, 0111 and 1111, 0001 and 0000,0100 and 0000 ) and three cases of high similarity between categories (1000 and 0000,1010 and 1011, 1111 and 1011).

Some of the subjects learned either one of these two category structures under standard instructions. Essentially, in standard instructions, subjects were told to learn which exemplars belonged to each of the two categories and were given no additional information. There were two competing predictions for the learning of the LS and NLS categories under standard instructions. Independent-cue models predicted that the LS categories should be the easiest to learn, because all members of the LS categories could be correctly classified if more weight was placed on the first three dimensions. In contrast, relational coding models predicted that the NLS categories should be the easiest to learn, because these categories were associated with more cases of high similarity within categories and fewer cases of high similarity between categories.

Of more importance was the learning of the LS and NLS task under theory instructions. These instructions provided subjects with two sets of facts. One set of facts provided information about some hypothetical birds, and the other set of facts provided information about some hypothetical bees. The facts about birds were that birds cannot see color, like to hover, cannot smell, and are active at night. The facts about bees were that bees could see color, like

Table 1

Linearly Separable Categories and Not Linearly Separable Categories

\begin{tabular}{|c|c|c|c|c|c|c|c|c|c|}
\hline \multicolumn{5}{|c|}{ Category A (Bird Flowers) } & \multicolumn{5}{|c|}{ Category B (Bee Flowers) } \\
\hline \multirow[b]{2}{*}{ Exemplars } & \multicolumn{4}{|c|}{ Dimensions } & \multirow[b]{2}{*}{ Exemplars } & \multicolumn{4}{|c|}{ Dimensions } \\
\hline & Di & D2 & D3 & D4 & & D1 & $\mathrm{D} 2$ & D3 & D4 \\
\hline \multicolumn{10}{|c|}{ Linearly Separable Categories } \\
\hline Al & 1 & 0 & 1 & 1 & B1 & 1 & 0 & 0 & 1 \\
\hline $\mathrm{A} 2$ & 1 & 0 & 1 & 0 & B2 & 0 & 0 & 1 & 0 \\
\hline A3 & 1 & 1 & 0 & 1 & B3 & 0 & 1 & 0 & 0 \\
\hline A4 & 0 & 1 & 1 & 0 & B4 & 0 & 0 & 0 & 1 \\
\hline \multicolumn{10}{|c|}{ Not Linearly Separable Categories } \\
\hline Al & 1 & 0 & 0 & 0 & B1 & 0 & 0 & 0 & 1 \\
\hline $\mathrm{A} 2$ & 1 & 0 & 1 & 0 & B2 & 0 & 1 & $\mathbf{0}$ & 0 \\
\hline A3 & 0 & 1 & 1 & 1 & B3 & 1 & 0 & 1 & 1 \\
\hline A4 & 1 & 1 & 1 & 1 & B4 & 0 & 0 & 0 & 0 \\
\hline
\end{tabular}


to land, can smell, and are active during the day. The subjects were explicitly told to use these facts in category learning, because one category would have flowers that tend to attract birds, and the other category would have flowers that tend to attract bees. Providing a theory might facilitate learning of the LS categories, because each set of facts was related to most of the attributes comprising the exemplars within an LS category. However, the theory instructions might not be expected to facilitate learning of the NLS categories, because each set of facts, corresponding to a category, was less related to the attributes comprising one of the exemplars (A1 and B3) in an NLS category.

\section{METHOD}

\section{Subjects}

Subjects were 64 male and female undergraduates at the University of Illinois. They participated in the experiment in order to partially fulfill an introductory psychology course requirement. These 64 subjects were randomly and evenly assigned to the four cells of the 2 instructional set (theory vs. standard instructions) $\times 2$ category structure (LS vs. NLS) between-subjects design. Three subjects' ( 1 in the Theory-NLS condition and 2 in the StandardNLS condition) partially collected data were discarded, because these subjects accidentally caused the computer to malfunction at some point during the experiment. As a consequence, data were collected from 3 new subjects.

\section{Stimuli and Apparatus}

The training stimuli were 16 verbal descriptions of hypothetical flowers, 8 of which belonged to the LS task and 8 of which belonged to the NLS task. The transfer tests consisted of the 8 training flower descriptions, comprising either the LS or NLS tasks, as well as 8 new flower descriptions for each of these two tasks. These 8 new descriptions, for each task, were generated by exhausting the remaining possible binary valued combinations across the four dimensions.

A microcomputer (Apple II)' connected to a television set controlled the presentation and recorded the subjects' category choices made in response to the training and transfer flower descriptions, which appeared on the television screen. The four attributes characterizing a given flower description were presented on the television screen $(13 \times 17 \mathrm{in}$.) as four short phrases printed in bold white capital letters, with each phrase appearing centered and subsumed directly below the preceding phrase.

\section{Procedure}

The experimental session consisted of instructional, initial learning, and transfer phases.

Instructions. Subjects participated on an individual basis. Two types of instructions (standard and theory) were employed. Standard instructions informed the subjects that they would be presented with eight different flower descriptions, each of which would be presented a number of times. They were told to learn to correctly classify the flower descriptions as belonging to either Category A or Category B. Finally, these subjects were informed that this learning task would initially appear difficult, but eventually the task would become easier, because corrective feedback would be given after each category choice.

The subjects who received the theory instructions were given the same instructions as subjects receiving standard instructions, except that they received additional information. These subjects were provided some specific facts about some hypothetical birds and bees. Specifically, they were explicitly told that they would be learning some facts about some hypothetical birds and bees and that they should use these facts to help them in learning the categories. Furthermore, theory instructions emphasized that flowers that tend to attract birds belong to one category, whereas flowers that tend to attract bees belong to the other category. Following these instructions, the subjects were given a sheet that stated that birds cannot see color, like to hover, cannot smell, and are active at night, whereas bees can see color, like to land, can smell, and are active during the day. The subjects were told to study these facts for a minute, after which time they would be asked to recall all eight facts. If a subject did not correctly recall the facts, he or she was asked to restudy them. The purpose of having the subjects show perfect recall for all of the facts was to ensure that the facts had been stored and would be equally available to help in learning the categories.

Learning. After all subjects received one of the two types of instructions, they began the initial learning phase. The subjects began to classify the training flower descriptions constituting either the LS or NLS task. The computer controlled the presentation and recorded the subject's category choice for each flower description. The training procedure involved presenting the flower descriptions one at a time. For a given flower-description presentation, the subject had as much time as needed to make a category choice. Upon choosing a category, the subject had to push either an A or B button located on the computer keyboard in order to indicate a category $A$ or B choice. After pushing the choice button, the subject then had to push another button and was given immediate feedback as to whether the category choice was correct or incorrect. Following the feedback, there was a 5-sec interstimulus interval with the flower description remaining on the screen before the next flower description appeared. This training procedure was consistently repeated until either the subject had received a maximum of 16 runs through the eight flower descriptions, or the subject met the learning criterion of two consecutive errorless runs. It should be noted that there was not an obvious break between the end of one run and the beginning of the next one. The interrun interval was held constant at $5 \mathrm{sec}$.

Appropriate counterbalancing of category labels, randomization of flower descriptions and their associated attributes was undertaken. For each category structure, half of the subjects received four flower descriptions (A1-A4) as belonging to Category A and the other four flower descriptions (B1-B4) as belonging to Category B. In contrast, the other half of the subjects received the opposite assignment of flower descriptions to category labels. Across runs, for each category structure, the presentation order of the eight exemplars was randomized. Also, the presentation order of the attributes comprising the eight flower descriptions was randomized across runs.

Transfer. After completing the learning phase, all subjects were given transfer tests. The transfer tests consisted of the eight training descriptions and eight new flower descriptions. All subjects were told that they would be classifying both old and new flower descriptions and that they should make their category judgments based on what they had learned. Also, they were informed that there would not be any corrective feedback given after making a category judgment.

The computer presented, via the television screen, the old and new flower descriptions one at a time. After a given flower description appeared on the screen, the subject had as much time as needed to determine a category judgment for the flower description in question. Upon reaching a judgment, the subject pushed either the $A$ or the B button in order to indicate a category A or B judgment. Immediately following the button press, the subject pushed another button to receive the next flower description. The interstimulus interval was $5 \mathrm{sec}$, and, during this time, the flower description was still displayed on the screen. This presentation procedure involved presenting each flower description at least once but not more than five times. A programming error resulted in varying the number of presentation trials for each of the transfer descriptions. However, this error did not seem to introduce any serious confoundings. A total of 32 flower description trials always resulted for each sub- 
ject. The presentation order of the flower descriptions and their associated attributes were randomized across trials. The entire experimental session lasted approximately 40-50 minutes.

\section{RESULTS}

\section{Errors During Initial Learning}

The errors during learning were analyzed by a threeway ANOVA. This analysis included the factors of instructional set (theory vs. standard instructions) and category structure (LS vs. NLS) as between-subjects factors and blocks (Runs $1-4$ vs. 5-8 vs. 9-12 vs. 13-16) as a within-subjects factor. For all of the analyses to be reported, the statistical level of significance was set at $p<.05$. The main effect for both instructional set and category structure were not significant (both $F s<1$ ). However, there was an important instructional set $x$ category structure interaction $[\mathrm{F}(1,60)=7.26$, MSe $=$ 83.51]. Simple main effects analyses were performed on category structure. Under theory instructions, the LS task $(M=31.13)$ resulted in significantly fewer errors than the NLS task $(\mathrm{M}=49.56)[\mathrm{F}(1,60)=32.57$, MSe $=$ 83.51]. Standard instructions did not lead to a difference in errors between the LS task ( $M=45.44)$ and NLS task $(\mathbf{M}=39.25), \mathrm{F}<1$. However, this difference was in the direction predicted by relational coding models. Also, simple main effects analyses were performed on instructional set. The LS task resulted in significantly fewer errors under theory instructions than under standard instructions $[\mathrm{F}(1,60)=19.62, \mathrm{MSe}=83.51]$. The NLS task resulted in significantly fewer errors under standard instructions than under theory instructions $[F(1,60)=$ $10.18, \mathrm{MSe}=83.51]$. There was a significant effect of blocks $[\mathrm{F}(3,180)=36.69$, MSe $=10.38]$. In addition, there was a significant blocks $\times$ category structure interaction $[\mathrm{F}(3,180)=3.71, \mathrm{MSe}=10.38]$. Neither the blocks $x$ instructional set interaction nor the blocks $X$ instructional set $x$ category structure interaction reached significance.

\section{Theoretical Modeling Analyses}

A mixed representational model was tested in order to investigate the nature of the category representations mediating classification performance during transfer. This model was fitted to the overall classification probabilities obtained in each of the four experimental conditions. The mixed model (Medin et al., 1984) has an exemplar component and a prototype component, in which each component specifies how a test probe should be classified based on its similarity to stored exemplar and prototypical information.

The exemplar component of the mixed model is conceptually and computationally the same as formulated in the context model (Medin \& Schaffer, 1978). According to the exemplar component, the evidence favoring classifying some test exemplar into a given category depends on how similar this exemplar is to the stored exemplars of one category versus the contrast category. Quantita- tively speaking, the probability of classifying a test probe into Category A (B) is equal to the sum of the similarities of the probe to each of the stored exemplars for that category divided by the sum of the similarities of the probe to all stored exemplars comprising both categories. The similarity between a test probe and any given exemplar is determined by multiplying the four parameters corresponding to each of the four category dimensions. Each parameter represents the degree of similarity between the two attribute values comprising a given dimension. The degree of similarity as represented in a parameter can take on any value between 0 and 1 . Zero as a parameter value suggests that the associated dimension is very salient and that each of the attribute values is distinctively encoded and may serve as a basis for classification. In contrast, a very large similarity parameter suggests that the dimension is not salient and its associated attribute values have not been distinctively encoded. As an example, the similarity between test probe 1010 and stored exemplar 0110 can be expressed as $\mathrm{D} 1 \times \mathrm{D} 2 \times 1 \times 1$, or simply as $\mathrm{D} 1 \times \mathrm{D} 2$. The similarity between the two exemplars is that they are maximally similar on dimensions D3 and D4, but different on dimensions D1 and D2.

The prototype component of the mixed model assumes that a given test probe can also be classified on the basis of how similar it is to one prototype versus the contrast prototype. A ratio rule involving similarities is employed and, in quantitative terms, the probability of classifying a probe into a category is determined by multiplying the parameter values associated with the four dimensions of the prototype of that category divided by the value obtained when this multiplicative rule is applied in determining the probe's similarity to both categories' respective prototypes. As with the exemplar component, the parameter values still range from 0 to 1 and they carry the same interpretative meaning. As an example, the similarity between test probe 1001 and prototype 0000 would be $\mathrm{D} 1 \times 1 \times 1 \times \mathrm{D} 4$, or simply $\mathrm{D} 1 \times \mathrm{D} 4$.

In addition, the mixed model has a fifth parameter that weights the degree to which classification is governed by the exemplar versus the prototype component. This fifth parameter, $T$, varies from 0 to 1 . $T$ is the probability of relying on prototypical information, and $1-\mathrm{T}$ is the probability of relying on exemplar information. A T of 0 or 1 indicates exclusive reliance on exemplar and prototypical information, respectively. A T of .50 would suggest roughly equal reliance on both types of information.

Classification equations based on the exemplar and prototype components were written for the old and new exemplars that were classified by each of the four groups during transfer. Marquardt's (1963) nonlinear least squares technique was employed to find the best fitting parameter estimates that would minimize the average $a b-$ solute deviation between the obtained and predicted classification probabilities.

Table 2 shows the observed and predicted classification probabilities for the theory-LS and standard-LS conditions. Table 3 shows these probabilities for the theory- 
Table 2

Transfer Classification Probabilities for Theory-LS and Standard-LS Conditions

\begin{tabular}{|c|c|c|}
\hline Exemplars & Theory-LS & Standard-LS \\
\hline Al 1011 & $.97 \quad(.96)$ & $.84 \quad(.82)$ \\
\hline A2 1010 & $.90 \quad(.94)$ & $.87 \quad(.82)$ \\
\hline A3 1101 & $.81 \quad(.83)$ & $.58 \quad(.62)$ \\
\hline A4 0110 & $.84 \quad(.85)$ & .62 (.61) \\
\hline BI 1001 & $.90 \quad(.85)$ & $.73 \quad(.62)$ \\
\hline B2 0010 & .67 (.65) & $.58 \quad(.60)$ \\
\hline B3 0100 & $.97 \quad(.95)$ & $.91 \quad(.80)$ \\
\hline B4 0001 & $1.00 \quad(.98)$ & $.80 \quad(.87)$ \\
\hline N1 1111 & $.93(.97)$ & $.80 \quad(.84)$ \\
\hline $\mathrm{N} 2 \quad 1100$ & $.49 \quad(.52)$ & $.56 \quad(.55)$ \\
\hline N3 0111 & $.86 \quad(.88)$ & $.59 \quad(.60)$ \\
\hline N4 1000 & $.11 \quad(.12)$ & $.41 \quad(.44)$ \\
\hline N5 0101 & $.03 \quad(.09)$ & $.21 \quad(.24)$ \\
\hline N6 0000 & $.18 \quad(.02)$ & $.38 \quad(.15)$ \\
\hline N7 0011 & $.48 \quad(.50)$ & $.33 \quad(.43)$ \\
\hline N8 $\quad 1110$ & $.94 \quad(.96)$ & $.85 \quad(.83)$ \\
\hline
\end{tabular}

Note-For training descriptions, classification probabilities were scored with respect to correct category. For new descriptions, classification probabilities were scored with respect to Category A. Predicted probabilities are in parentheses.

Table 3

Transfer Classification Probabilities for Theory-NLS and Standard-NLS Conditions

\begin{tabular}{|c|c|c|c|c|c|}
\hline \multicolumn{2}{|c|}{ Exemplars } & \multicolumn{2}{|c|}{ Theory-NLS } & \multicolumn{2}{|c|}{ Standard-NLS } \\
\hline$\overline{A l}$ & 1000 & .36 & $(.41)$ & .33 & $(.47)$ \\
\hline A2 & 1010 & .74 & $(.71)$ & .97 & $(.85)$ \\
\hline A3 & 0111 & .78 & (.75) & .79 & (.96) \\
\hline A4 & 1111 & .76 & $(.85)$ & 1.00 & $(.91)$ \\
\hline B1 & 0001 & .88 & $(.82)$ & .89 & $(.98)$ \\
\hline B2 & 0100 & .74 & $(.90)$ & 1.00 & $(99)$ \\
\hline B3 & 1011 & .30 & (.23) & .48 & $(.36)$ \\
\hline B4 & 0000 & .72 & $(.91)$ & .96 & (.99) \\
\hline NI & 1100 & .50 & $(.50)$ & .63 & (.61) \\
\hline $\mathrm{N} 2$ & 0010 & .17 & (.26) & .21 & $(.11)$ \\
\hline N3 & 0101 & .31 & (.32) & .32 & $(.26)$ \\
\hline N4 & 0011 & .58 & $(.57)$ & .63 & $(.64)$ \\
\hline N5 & 1110 & .76 & (.63) & .95 & $(.86)$ \\
\hline N6 & 1101 & .62 & $(.74)$ & .73 & $(.89)$ \\
\hline N7 & 0110 & .32 & $(.36)$ & .13 & $(.15)$ \\
\hline N8 & 1001 & .69 & $(.62)$ & .69 & $(.67)$ \\
\hline
\end{tabular}

Note-For training descriptions, classification probabilities were scored with respect to correct category. For new descriptions, classification probabilities were scored with respect to Category $A$. Predicted probabilities are shown in parentheses.
NLS and standard-NLS conditions. For each of the four conditions, there was a reasonably close fit between the predicted and obtained classification probabilities. The mixed model accounted for $98 \%, 82 \%, 85 \%$, and $89 \%$ of the variance in the transfer probabilities for the theoryLS, theory-NLS, standard-LS, and standard-NLS conditions, respectively.

Table 4 shows the model's best-fitting parameter values and associated standard errors, average absolute deviations, and sum of squared deviations for all four experimental conditions. For the most part, the four conditions showed small standard errors associated with their respective parameter values. However, the $T$ parameter for the theory-LS condition and the D4 parameter for the standard-LS condition revealed large standard errors. Thus, interpretative caution is warranted for these particular parameter estimates, because they may be quite unstable. Notice that the parameter $\mathrm{T}$ is of intermediate value $(.51-.58)$ for the theory-LS, theory-NLS, and standard-NLS conditions. Thus, it appears that subjects in these three conditions were relying on both exemplar and prototypical information in classification. In contrast, notice that for the standard-LS condition, the parameter $\mathrm{T}$ is 0 , which suggests that there was exclusive reliance on exemplar level information in classification.

\section{DISCUSSION}

An important finding emerged, which was that theory instructions led to substantially fewer errors during the learning of the LS categories. There is now tentative evidence that classification is better for LS categories when the theory's facts are related to most of the attributes comprising the exemplars. The initial source of the theory's ability to facilitate the learning of the LS categories can be viewed in terms of the theory's influence on the storage of different levels of category information, which was in turn mediating classification. The mixed-model fitting of the transfer data suggested that there was storage and reliance on both prototypical and exemplar level information in the theory-LS condition. In comparison, the mixedmodel fitting of the transfer data for the standard-LS condition suggested that there was storage and reliance only

Table 4

Critical Values for the Mixed-Model Fitting of Transfer Probabilities in Each Condition

\begin{tabular}{|c|c|c|c|c|}
\hline $\begin{array}{c}\text { Parameter } \\
\text { Values }\end{array}$ & Theory-LS & Standard-LS & Theory-NLS & Standard-NLS \\
\hline Dl & $.14 \quad(.09)$ & $.22 \quad(.07)$ & $.28 \quad(.08)$ & $.03 \quad(.02)$ \\
\hline D2 & $.25 \quad(.08)$ & $.50 \quad(.14)$ & $.63(.16)$ & $.26 \quad(.16)$ \\
\hline D3 & $.04 \quad(.06)$ & $.21 \quad(.08)$ & $.37(.10)$ & $.11(.07)$ \\
\hline D4 & $.61 \quad(.28)$ & $.42 \quad(.44)$ & $.32(.14)$ & $.04 \quad(.03)$ \\
\hline $\mathrm{T}$ & $.51 \quad(.51)$ & $.00 \quad(.18)$ & $.58 \quad(.13)$ & $.53 \quad(.08)$ \\
\hline $\begin{array}{c}\text { Average Absolute } \\
\text { Deviations } \\
\text { Sum of Squared }\end{array}$ & .03 & .06 & .07 & .08 \\
\hline Deviations & .04 & .10 & .13 & .14 \\
\hline
\end{tabular}

Note-Standard errors for parameter values are in parentheses. 
on exemplar information. Also, there is preliminary evidence that a theory will not help and may impair the learning of NLS categories when there are several exemplars whose attributes are unrelated to the theory's facts.

The present study is similar in certain respects to a study by Wattenmaker et al. (1984). These investigators found that the learning of LS categories was facilitated by prior knowledge, whereas prior knowledge interfered with the learning of NLS categories. This facilitative and interfering effect of prior knowledge on category learning occurred under two knowledge inducing conditions. In one condition, the category exemplars spontaneously activated schema-based prior knowledge, which in turn guided category learning. In the other condition, instructions were given that served to activate theme-based or theory-based prior knowledge, which in turn guided category learning. Thus, category learning is affected by inducing various types of prior knowledge (Wattenmaker et al., 1984) or by providing new knowledge as was the case in the present study.

In the area of classification learning, there have been insightful discussions on the role of knowledge in classification learning (Carey, 1982; Medin \& Smith, 1984; Murphy \& Medin, 1985; Wattenmaker et al., 1984). Future work in classification learning might benefit from further attempts to interface it with research on higher order knowledge representation (Anderson, 1976; Anderson \& Bower, 1973; Brewer \& Pani, 1983; Gentner \& Stevens, 1983; Graesser \& Clark, 1985; Johnson-Laird, 1983; Kintsch, 1974; Norman \& Rumelhart, 1975; Schank, 1982).

In closing, the theory's ability to help in learning exemplars whose attribute values were related to the theory's facts adds to research findings arising in other research areas investigating various aspects of the cognitive system. In various research areas, it has been shown that appropriate knowledge facilitates the acquisition of information that is related to it. For example, appropriate prior knowledge facilitates comprehension of paragraphs (Roth \& Shoben, 1983), memory for places (Brewer \& Treyens, 1981), memory for narrative prose (Anderson \& Pichert, 1978; Bower, 1976; Bransford \& Johnson, 1973; Lichtenstein \& Brewer, 1980), and memory for transfer passages (Thorndyke, 1977). Furthermore, in other areas, facilitative effects of relevant prior knowledge or recently acquired relevant knowledge are found in perceptual identification of objects (Biederman, Glass, \& Stacy, 1973; Palmer, 1975), in verifying inferences about famous people (Clifton \& Slowiaczek, 1981 ), in generating the names of categories (Barsalou, 1983), in making a judgment (Srull \& Wyer, 1980; Tversky \& Kahneman, 1973), in solving a problem (Gick \& Holyoak, 1983), and in learning a cognitive skill (Ross, 1984).

\section{REFERENCES}

ANDERSON, J. R. (1976). Language, memory, and thought. Hillsdale, NJ: Erlbaum.
ANDERSON, J. R., \& Bower, G. H. (1973). Human associative memory. Washington, DC: Winston.

Anderson, R. C., \& PicherT, J. W. (1978). Recall of previously unrecallable information following a shift in perspective. Joumal of Verbal Learning \& Verbal Behavior, 17, 1-12.

Barsalou, L. W. (1983). Ad hoc categories. Memory \& Cognition, 11, $211-227$.

Biederman, I., Glass, A. L., \& Stacy, E. W., JR. (1973). Searching for objects in real-world scenes. Joumal of Experimental Psychology, 97, 22-27.

BoURNe, L. E. (1966). Human conceptual behavior. Boston: Allyn \& Bacon.

BoWER, G. H. (1976). Experiments on story understanding and recall. Quarterly Journal of Experimental Psychology, 28, 511-534.

BRANSFord, J. D., \& Johnson, M. K. (1973). Considerations of some problems of comprehension. In W. G. Chase (Ed.), Visual information processing. New York: Academic Press.

BREWER, W. F., \& PANI, J. R. (1983). The structure of human memory. In G. H. Bower (Ed.), The psychology of learning and motivation: Advances in research and theory (Vol. 17). New York: Academic Press.

BREWER, W. F., \& TREYeNS, J. C. (1981). Role of schemata in memory for places. Cognitive Psychology, 13, 207-230.

BRooKS, L. (1978). Nonanalytic concept formation and memory for instances. In E. Rosch \& B. B. Lloyd (Eds.), Cognition and categorization. Hillsdale, NJ: Erlbaum.

CAREY, S. (1982). Semantic development: The state of the art. In E. Wanner \& L. R. Gleitman (Eds.), Language acquisition: The state of the art. New York: Cambridge University Press.

Clifton, C., JR., \& SlowiaczeK, M. L. (1981). Integrating new information with old knowledge. Memory \& Cognition, 9, 142-148.

Elio, R., \& ANDERSON, J. R. (1981). The effects of category generalizations and instance similarity on schema abstraction. Journal of Experimental Psychology: Human Learning \& Memory, 7, 397-417.

FRANKS, J. J., \& BRANSFORD, J. D. (1971). Abstraction of visual patterns. Journal of Experimental Psychology, 90, 65-74.

Gentner, D., \& Stevens, A. L. (1983). Mental models. Hillsdale, NJ: Erlbaum.

GICK, M. L., \& HOLYOAK, K. J. (1983). Schema induction and analogical transfer. Cognitive Psychology, 15, 1-38.

Graesser, A. C., Clark, L. F. (1985). The structures and procedures of implicit knowledge. Norwood, NJ: Ablex.

Hintzman, D. L., \& LudiaM, G. (1980). Differential forgetting of prototypes and old instances: Simulation by an exemplar-based classification model. Memory \& Cognition, 8, 378-382.

Homa, D., \& VosburGH, R. (1976). Category breadth and the abstraction of prototypical information. Journal of Experimental Psychology: Human Learning \& Memory, 2, 322-330.

JohnSon-Laird, P. N. (1983). Mental models. Cambridge, MA: Harvard University Press

KINTSCH, W. (1974). The representation of meaning in memory. Hillsdale, NJ: Erlbaum.

Lichtenstern, E. H., \& Brewer, W. F. (1980). Memory for goaldirected events. Cognitive Psychology, 12, 412-445.

MARQUARDT, D. W. (1963). An algorithm for least-squares estimation of nonlinear parameters. Journal of the Society for Industrial \& Applied Mathematics, 11, 431-441.

MCCloskey, M. E., \& GlucksBerg, S. (1978). Natural categories: Well defined or fuzzy sets? Memory \& Cognition, 6, 462-472.

MEdin, D. L. (1983). Structural principles of categorization. In B. Shepp $\& \mathrm{~T}$. Tighe (Eds.), Interaction: Perception, development, and cognition. Hillsdale, NJ: Erlbaum.

Medin, D. L.. Altom, M. W., Edelson, S. M., \& Freko, D. (1982). Correlated symptoms and simulated medical classification. Journal of Experimental Psychology: Learning, Memory, \& Cognition, 8, 37-50.

Medin, D. L., Altom, M. W., \& Murphy, T. D. (1984). Given versus induced category representations: Use of prototype and exemplar information in classification. Journal of Experimental Psychology: Leaming, Memory, \& Cognition, 10, 333-352.

Medin. D. L.. Dewey, G. I. \& Murphy, T. D. (1983). Relationships between item and category learning: Evidence that abstraction is not 
automatic. Journal of Experimental Psychology: Learning, Memory, \& Cognition, 9, 607-625.

Medin, D. L., \& Schaffer, M. M. (1978). Context theory of classification learning. Psychological Review, 85, 207-238.

Medin, D. L., \& SChWANENFlugel, P. J. (1981). Linear separability in classification learning. Joumal of Experimental Psychology: $\mathrm{Hu}$ man Learning \& Memory, 7, 355-368.

MEDIN, D. L., \& SMITH, E. E. (1981). Strategies and classification learning. Journal of Experimental Psychology: Human Learning \& Memory, 7, 241-253.

Medin, D. L., \& Smith, E. E. (1984). Concepts and concept formation. In M. R. Rosenweig \& L. W. Porter (Eds.), Annual Review of Psychology, 35, 113-138.

MuRPhy, G. L., \& MEdin, D. L. (1985). The role of theories in conceptual coherence. Psychological Review, 92, 289-316.

Norman, D. A., \& RumelharT, D. E. (1975). Explorations in cognition. San Francisco: Freeman.

Palmer, S. E. (1975). The effects of contextual scenes on the identification of objects. Memory \& Cognition, 3, 519-526.

POSNER, M. I., \& KEELE, S. W. (1968). On the genesis of abstract ideas. Journal of Experimental Psychology, 77, 353-363.

ReEd, S. K. (1972). Pattern recognition and categorization. Cognitive Psychology, 3, 382-407.

ReIrMAN, J. S., BowER, G. H. (1973). Storage and later recognition of exemplars of concepts. Cognitive Psychology, 4, 194-206.

Rosch, E. (1975). Cognitive representations of semantic categories. Journal of Experimental Psychology: General, 104, 192-233.

Rosch, E., \& MERvis, C. B. (1975). Family resemblances: Studies in the internal structure of categories. Cognitive Psychology, 7, 573-605.
Ross, B. H. (1984). Remindings and their effects in learning a cognitive skill. Cognitive Psychology, 16, 371-416.

Roth, E. M., \& Shoben, E. J. (1983). The effect of context on the structure of categories. Cognitive Psychology, 15, 346-378.

Schank, R. C. (1982). Dynamic memory: A theory of reminding and learning in computers and people. Cambridge, England: Cambridge University Press.

Smith, E. E., \& Medin, D. L. (1981). Categories and concepts. Cambridge, MA: Harvard University Press.

SMITH, E. E., SHOBEN, E. J., \& RIPS, L. J. (1974). Structure and process in semantic memory: A featural model for semantic decisions. Psychological Review, 81, 214-241.

SRULL, T. K., \& WYER, R. S. (1980). Category accessibility and social perception: Some implications for the study of person memory and interpersonal judgments. Journal of Personality \& Social Psychology, 38, 841-856.

THORNDYKE, P. W. (1977). Cognitive structures in comprehension and memory of narrative discourse. Cognitive Psychology, 9, 77-110.

TVERSKY, A., \& KAHNEMAN, D. (1973). Availability: A heuristic for judging frequency and probability. Cognitive Psychology, 5, 207-232.

Wattenmaker, W. D., Dewey, G. I., Murphy, T. D., \& Medin, D. L. (1984). Linear separability and concept learning: Context, relational properties, and concept naturalness. Manuscript submitted for publication.

(Manuscript received January 9, 1984; revision accepted for publication June 5,1985 .) 